\title{
Dynamic distribution of TTK in HeLa cells: insights from an ultrastructural study
}

\author{
Zhen DOU ${ }^{1 *}$, Akira SAWAGECHI ${ }^{2,}{ }^{*}$, Jie ZHANG ${ }^{1}$, Hong LUO ${ }^{1}$, LAwrence BRAKO ${ }^{3}$, Xue Biao YAO ${ }^{1,2, * *}$ \\ ${ }^{1}$ Laboratory of Cell Dynamics, School of Life Sciences, University of Science and Technology of China, \\ Hefei, 230027,ChinaE-mail: yaoxb@ustc.edu.cn \\ ${ }^{2}$ Department of Molecular and Cell Biology, University of California, Berkeley, CA 94720, USA \\ ${ }^{3}$ Electron Microscopy Facility, Morhouse School of Medicine, Atlanta, GA 30310, USA
}

\begin{abstract}
Entry into mitosis is driven by signaling cascades of mitotic kinases. Our recent studies show that TTK, a kinetochore-associated protein kinase, interacts with CENP-E, a mitotic kinesin located to corona fiber of kinetochore. Using immunoelectron microscopy, here we show that TTK is present at the nuclear pore adjacent complex of interphase HeLa cells. Upon nuclear envelope fragmentation, TTK targets to the outermost region of the developing kinetochores of monoorient chromosome as well as to spindle poles. After stable attachment, throughout chromosome congression, TTK is a constituent of the corona fibers, extending up to $90 \mathrm{~nm}$ away from the kinetochore outer plate. Upon metaphase alignment, TTK departs from the kinetochore and migrates toward the centrosomes. Taken together, this evidence strongly supports a model in which TTK functions in spindle checkpoint signaling cascades at both kinetochore and centrosome.
\end{abstract}

Ked words: mitosis, kinetochore, TTK.

\section{INTRODUCTION}

Chromosome movements during mitosis are orchestrated by the interaction of spindle microtubules with a specialized chromosome domain located within the centromere [1]. This specialized proteinous region, called the kinetochore $[2,3]$, is responsible for mitotic microtubule-centromere association. Structurally, the kinetochore is composed of four layers: an innermost plate that apparently consists of a specialized layer of chromatin, an interzone, an outer plate that has been argued to consist of tightly packed fibers $[4,5]$, and an outermost fuzzy, fibrous corona that is most clearly seen after microtubule disassembly [6, 7]. Although kinetochore morphology has been documented in numerous ultrastructural studies [8-12], there is little information about molecular compositionand the respective localization of known kinetochore proteins or protein complexes, except for a handful kinetochore proteins such as CENP-A (attached to

\footnotetext{
"These two authors contributed equally to this work

*** correspondence author: Xue Biao YAO, PhD, Laboratory of Cell Dynamics, School of Life Sciences, University of Science and Technology of China, Hefei 230027, China Tel: (551) 360-7141, Fax: (551) 360-7141, E-mail: yaoxb@ustc.edu.cn Received Jul-1 -2003 Revised Aug-11-2003 Accepted Sep-13-2003
}

to centromeric heterochromatin)[13], CENP-B (underneath the inner plate)[14], CENP-C (a component of the inner plate[1], CENP-E [a component of the corona fiber)[ 7], CENP-F (a component of outer plate)[15], Bub1 and BubR1 [components of the corona fiber)[16].

Recent studies reveal that CENP-E associates with spindle checkpoint kinases TTK[17-18] and BubR1[7]. TTK is a mammalian homologue of conserved Mps1 family members that are expressed in all proliferating cells and tissues[19, 20], consistent with its function in cell cycle progression. In all these Mps1 family members, the C-terminal catalytic domains show a high degree of sequence similarity[21]. Furthermore, both yeast and mammalian Mps1 kinases phosphorylate serine/threonine as well as tyrosine residues, at least in vitro[19, 22, 23]. However, the $\mathrm{N}$-terminal domains carry great sequence divergence. It was recently shown that amphibian Mps 1 is necessary for the establishment and maintenance of a spindle assembly checkpoint recon- stituted in Xenopus egg extracts[24], whereas murine Mps1 was reported to function in centrosome duplication[21]. However, it is unclear whether the outcomes reflect the difference between the two experimental systems or simply show 
the two sides of the same coin.

To better address the function of TTK in human cells, we have undertaken an immuno-electron microscopic analysis of human homologue MPS1, TTK in HeLa cells. We show that TTK has a dynamic distribution profile across cell cycle. In interphase cells, TTK is localized in nucleus adjacent to nuclear pore. Upon entering into mitosis, TTK becomes associated with the kinetochore's outermost region, corona fibers. In addition, TTK appears to traffic towards the centrosome associated with the minus-end directed motility along the mitotic spindle microtubules. TTK is released from the kinetochore as chromosomes align at the equator of the spindle and relocated to the spindle poles. Thus, TTK is a dynamic component of the fiberous corona, which monitors spindle checkpoint signaling and may provide a functional link between kinetochores and centrosomes in mitosis.

\section{MATERIALS AND METHODS}

\section{Antibodies}

An affinity-purified rabbit antibody against TTK (C-19; SC-540) was purchased from Santa Cruz Biotechnology (Santa Cruz, CA) while CENP-E antibody was produced as described previously[7].

To test the specificity of the TTK antibody, interphase and mitotic HeLa cells were harvested by sedimentation and lysed in RIPA buffer (25 m $M$ Tris-HCl, pH 7.5, $5 \mathrm{~m} M$ EDTA, 0.5\% SDS, and 1\% deoxycholate). The extracts were then sonicated and centrifuged to remove the residual insoluble materials. To test the subcellular distribution of TTK, chromosomal fraction from mitotic cells and nuclear fraction from interphase were also prepared[7]. Before electrophoresis, an appropriate amount of extract was diluted with $4 \mathrm{x}$ sample buffer and boiled for $2 \mathrm{~min}$. After separation in SDS-PAGE, the proteins were transferred onto a nitrocellulose membrane (Micron Separation Inc., Westborough, MA) and incubated with anti-TTK antibody and detected using ECL (Pierce, IL). Immunoreactive signals were visualized by autoradiography on Kodak BioMAX MR film (Rochester, NY).

\section{Cell culture}

HeLa cells, from American Type Culture Collection (Rockville, MD), were maintained as subconfluent monolayers in DMEMmedia (Invitrogen, Carlsbad, CA) with 10\% FBS (Hyclone, Inc. UT) and $100 \mathrm{U} / \mathrm{ml}$ penicillin plus $100 \mu \mathrm{g} / \mathrm{ml}$ streptomycin (Invitrogen, Carlsbad, CA)

\section{Immuno-electron microscopy}

For immunolabeling, cells were trypsinized and seeded onto acidtreated sterile 18-mm coverslips in six-well dishes (Corning Glass Works, Corning, NY). After reaching $75 \%$ confluence in $\sim 36 \mathrm{~h}$, cells were rinsed for $1 \mathrm{~min}$ with PHEM buffer (100 $\mathrm{m} M$ Pipes, $20 \mathrm{mM}$ Hepes, $\mathrm{pH}$ 6.9, $5 \mathrm{~m} M$ EGTA, $2 \mathrm{mM} \mathrm{MgCl}_{2}$, and $4 \mathrm{M}$ glycerol) and permeabilized for $1 \mathrm{~min}$ with PHEM plus $1 \%$ digitonin modified according to Yao et al[7]. Digitonin was selected to preferentially permeabilized plasma membrane to allow the egress of cytosolic proteins. Extracted cells were then fixed in $4 \%$ freshly made paraformaldehyde (Polysciences, Inc., Warrington, PA) plus 0.05\% glutaraldehyde (Tousimis Research Corp., Rockville, MD) in PHEM and rinsed three times in PBS. The coverslips were blocked with $0.05 \%$ Tween-20 in PBS (TPBS) containing 1\% BSA (Sigma Chemical Co.). The cells were incubated with TTK antibody in a humidified chamber for $1 \mathrm{~h}$ followed by three washes of TPBS. Visualization of TTK location was achieved by $10-\mathrm{nm}$ colloidal gold conjugated secondary antibody by modification of the protocol described by Yao et al[7, 25]. Basically, coverslips processed as above were rinsed with TPBS (3x5 min) and fixed with $1 \%$ glutaraldehyde (Tousimis) in PBS followed by three washes in PBS. Cells were then post-fixed in $2 \%$ osmium tetroxide (Electron Microscopy Sciences, Fort Washington, PA), dehydrated in a graded alcohol series followed by $100 \%$ acetone, and embedded in Epoxy (Ernest F. Fullam, Inc., Latham, NY). The cells were detached from coverslips using hydrofluoric acid, and the designated areas were excised and glued to blocks. Thin serial sections (silver-gold) were then cut, placed on copper grids, and stained with uranyl acetate and lead citrate. The sections were examined under a JEOL 1200 electron microscopy (Peabody, MA).

\section{RESULTS}

\section{TTK is associated with isolated mitotic chromo- somal fraction and interphase nuclei}

Our previous studies show that TTK is co-purified with CENP-E from mitotic HeLa cells while exogenous GFP-TTK is targeted to the kinetochore[17]. However, several recent studies have raised debates concerning the function and precise localization of TTK in mammalian cells[18, 21]. To define more closely the localization of TTK in the mitotic spindle, we carried out immuno-electron microscopic analysis using an affinitypurified antibody (C-19). The protein immunoblot analysis revealed that the TTK antibody specifically recognized a single protein band of $97 \mathrm{kD}$ in interphase and mitotic HeLa cell extracts as well as isolated chromosome and nuclear fractions (Fig 1A). The TTK protein abundance in interphase cell lysates is comparable to that of mitotic cells, indicating the expression of TTK protein is not tightly coupled with cell cycle regulation. Interestingly, TTK is enriched in nuclear fraction isolated from interphase HeLa cells and chromosome fraction of mitotic HeLa cells. In contrast, mitotic motor CENP-E is enriched in mitotic chromosome fraction but not nuclear fraction from interphase cells. Thus, we conclude that TTK is a nuclear-associated protein in interphase cells and associated with chromosomes of mitotic cells.

To examine precise TTK localization in interphase cells, we carried out immuno-electron microscopy on a HeLa cell in late G2 phase with apparent intact nuclear envelope (Fig 1B a). Examination of gold particles re- 

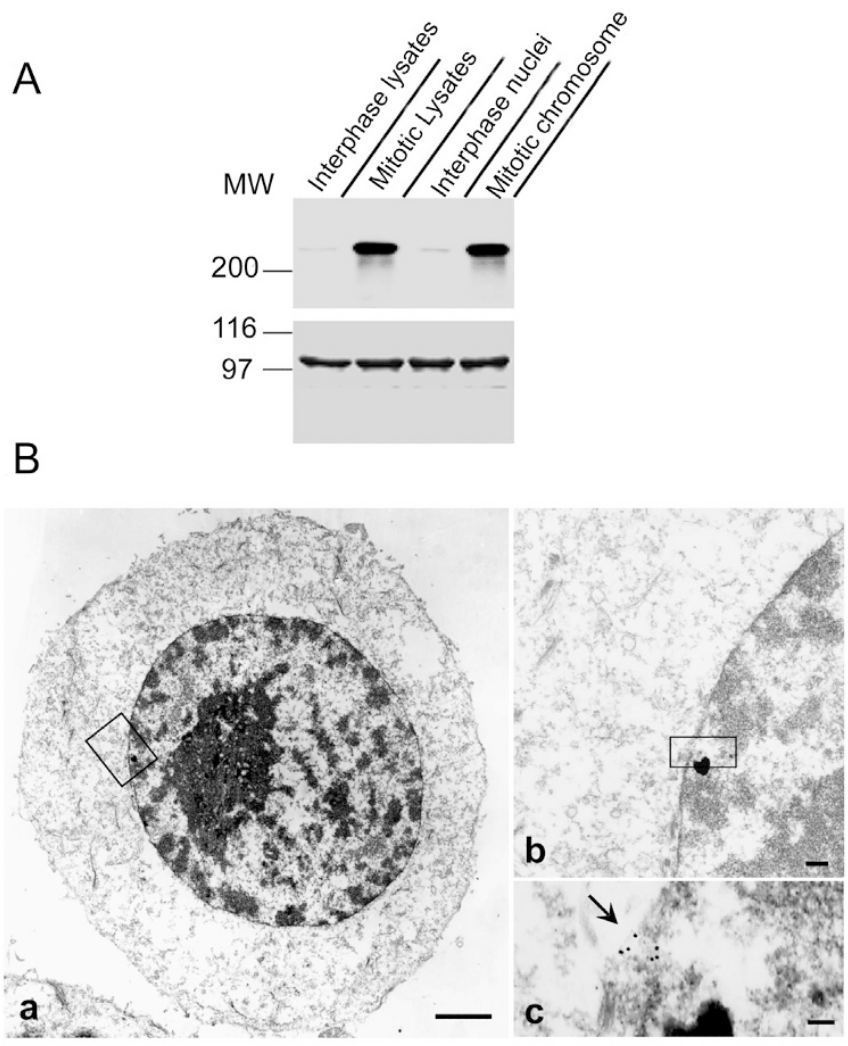

Fig 1. TTK is localized to nuclear pore of interphase HeLa cells. (A) Specificity of affinity purified TTK antibody. One immunoblot of interphase and mitotic whole cell lysates ( $50 \mu \mathrm{g} / \mathrm{lane})$, isolated interphase nuclei and mitotic chromosomes $(10 \mu \mathrm{g} / \mathrm{lane})$ separated in SDS-PAGE and transferred to a nitrocellulose membrane was probed with an affinity-purified TTK antibody. Note: The protein level of TTK is relatively unchanged between mitotic and interphase cell lysates while it is enriched in purified chromosome fraction. (B) TTK is localized to the nuclear pore of interphase HeLa cell. HeLa cells were processed as described in Materials and Methods. (a). Low magnification of an interphase HeLa cell with intact nuclear envelope. (b). Magnified view of boxed region in $a$ shows a nuclear pore. (c). Higher magnification view reveals that TTK is associated with nuclear pore complex. Arrow points to nuclear pore and adjacent six 10-nm gold particles. Bars: (a) $2 \mu \mathrm{m}$, (b) $200 \mathrm{~nm}$, (c) $100 \mathrm{~nm}$.

presenting the labeling of TTK revealed that the TTK is localized at the nuclear envelope adjacent to the nuclear pore complex (Fig 1B a-c). Careful examination of serial sections did not reveal any gold labeling inside the nucleus, indicating that TTK is a nuclear pore protein in interphase cells. Virtually no gold particles were found inside the other structures (i.e., vesicular membranes). These findings indicated that TTK is localized near the nuclear pore complex in interphase cells. Thus, we conclude that TTK is a nuclear pore protein component ininterphase cells and is associated with chromosome of mitotic cells, which is consistent with the outcome from an immunoflourescence study recently reported by Liu et al[26].

\section{TTK is localized to kinetochore and centrosome in prometaphase cells}

In the prometaphase, astral microtubules emanate from centrioles, coming in close proximity to newly condensing chromosomes to form bipolar spindle with two sets of centrioles sitting at opposite poles (Fig 2 a). As mono-orient chromosomes moved toward the pole, we examined TTK localization on the mono-oriented chromosomes in prometaphase cells. At magnified view, several gold particles representing specific labeling of TTK could clearly be seen on one leading kinetochore (i.e., defined here to be the one closer to the pole; Fig 2 b, arrow). Magnified view of boxed area Fig $2 b$ shows that TTK, marked by seven $10 \mathrm{~nm}$ gold particles is located to the outermost surface of the kinetochore (Fig $2 \mathrm{~d}$, arrow). Examination of gold particle labeling of another chromosome from same cells revealed that TTK is localized to the corona fibers of kinetochore of congressional chromosome (Fig 2c, arrows). Again, there was virtually no gold staining on other surface regions of the chromosome, indicating the specific association of TTK with kinetochore. A survey of gold labeling on 17 kinetochores indicates that TTK extends about $67 \mathrm{~nm}$ $(67 \pm 17 \mathrm{~nm}, \mathrm{n}=17)$ from the outer plate.

In addition, there are numerous gold particles adjacently located to spindle microtubule near the centrosomes (Fig 2b, arrowheads). Careful examination of the opposite centrosome also revealed that TTK localization adjacent to centrosome but not centrioles, indicating that TTK is also a centrosomal protein. Interestingly, substantial gold particles are also found in protein complex ( $\sim 50 \mathrm{~nm}$ in diameter, Fig $2 \mathrm{e}$ arrow) associated with spindle microtubules. It is possible that TTK is associated with a minus-end directed microtubule-based motor for its poleward trafficking and distribution. Thus, we concluded that TTK is located at both kinetochore and centrosomal region of prometaphase HeLa cells.

\section{At metaphase TTK departs from the kinetochore and traffics toward the spindle poles}

HeLa cells with aligned chromosomes were examined (Fig 3 a) to track TTK at bioriented kinetochores 


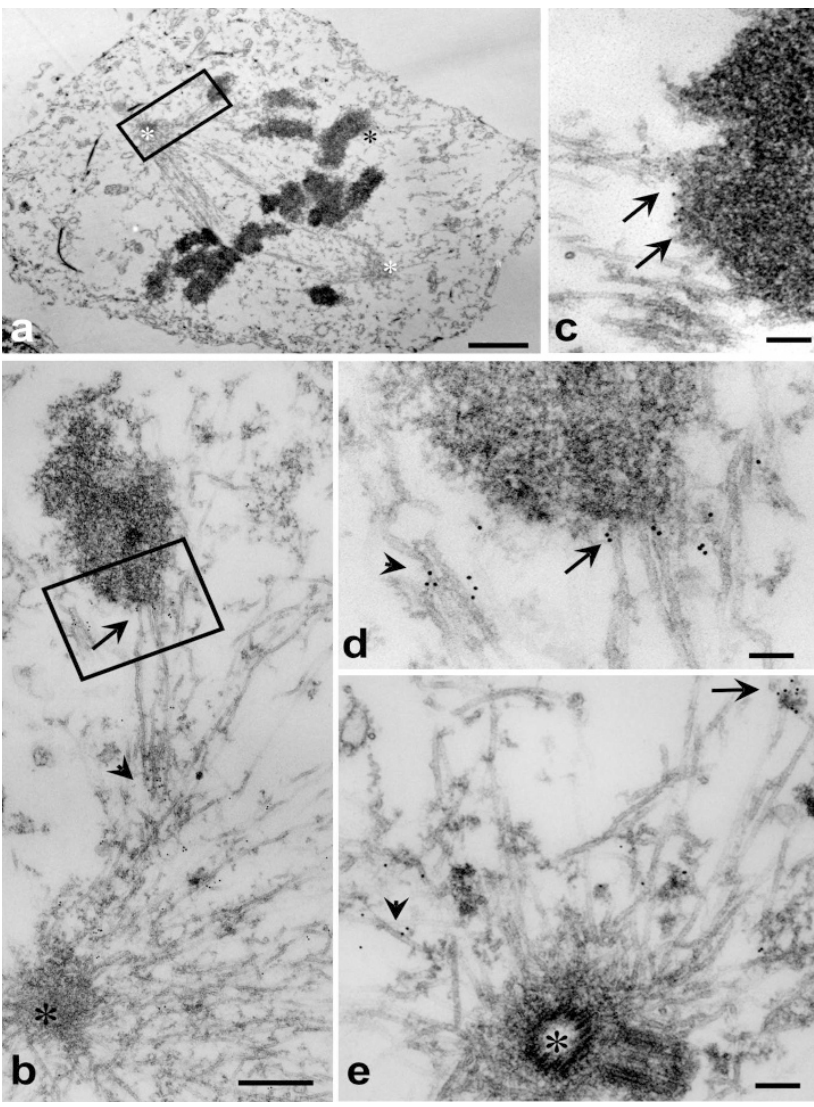

Fig 2. TTK is localized at the developing kinetochore and spindle poles of mitotic HeLa cells. HeLa cells grown on coverslips were pre-extracted and fixed. Visualization of TTK is achieved by $10 \mathrm{~nm}$ gold conjugated goat anti-rabbit IgG. (a) Low magnification of a prometaphase HeLa cells. Asterisks mark the two spindle poles of the bipolar spindle. An apparent mono-oriented chromosome is boxed, and higher magnification is shown in $\mathbf{b}$. (b) Magnified view of shows that $10 \mathrm{~nm}$ particles represent the TTK localization at the kinetochore (arrow) and spindle microtubules adjacent to centrosome (arrowhead). (c) Enlarged view of $\boldsymbol{b}$ shows a bi-oriented chromosome with $10 \mathrm{~nm}$ gold particles deposit onto the kinetochore (arrows). (d) Higher magnification view of boxed area of $\boldsymbol{b}$ shows that $10 \mathrm{~nm}$ gold particles decorate to the outer surface of the kinetochore interfaced with spindle microtubule (arrow). In addition, six $10 \mathrm{~nm}$ gold particles also mark an apparent protein complex associated with spindle microtubules (arrowhead). (e) Magnified view of a centrosome shows that a microtubule-associated protein complex decorated by eight 10-nm gold particles (arrow) traffics toward the pole. Several 10-nm gold particles (arrowhead) are also deposited to the pole 300 400 nm away from the centrioles. Bars: (a) $2 \mu \mathrm{m}$, (b) $500 \mathrm{~nm}$; (c-e) $150 \mathrm{~nm}$.

that had completed congression but were still under tentionexerted by opposing spindle microtubules[7]. Higher magnification views revealed that much fewer (Fig $3 \mathrm{~b}$, arrowheads) or virtually no gold particles surrounded the kinetochore (Fig $3 \mathrm{~b}$ and c, arrows). However,

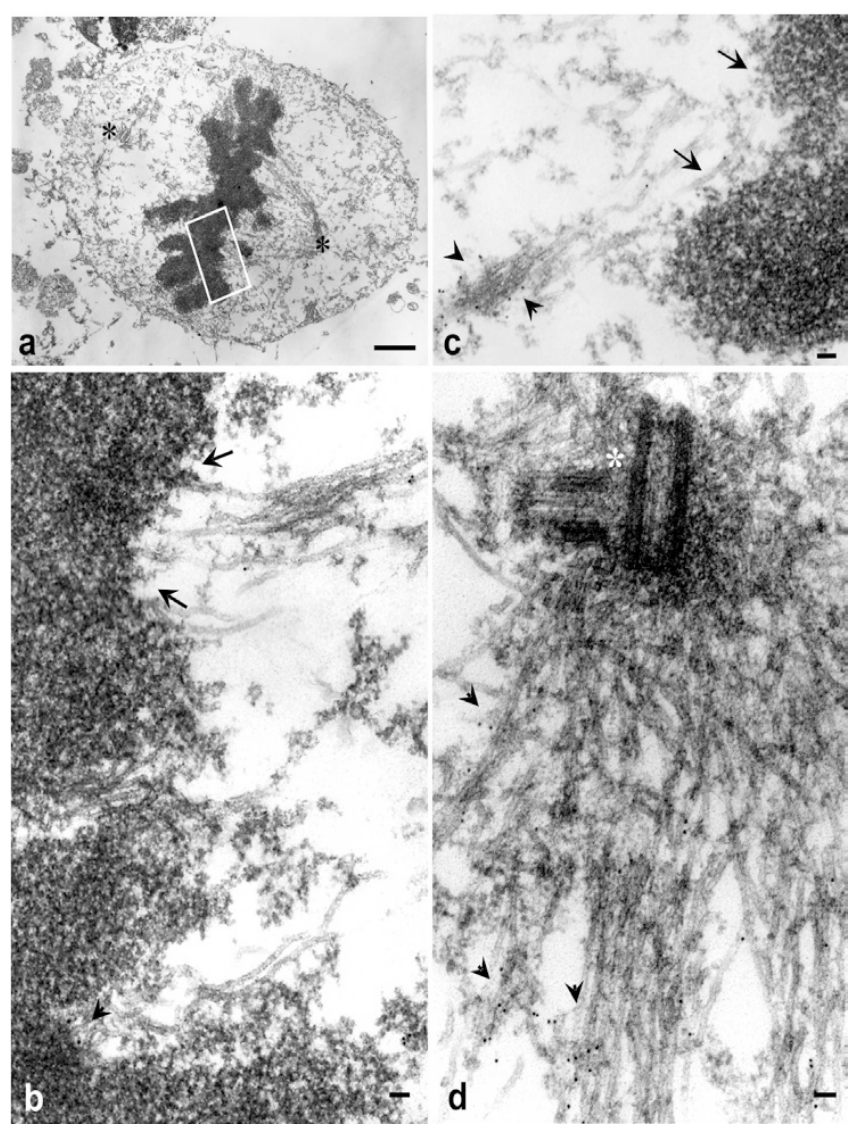

Fig 3. Upon the metaphase alignment, TTK departs from inetochores. HeLa cells were processed as described in Materials and Methods. (a) Low magnification view of a metaphase HeLa cell with chromosome aligned at the equator between the two spindle poles (asterisks). (b) Magnified view of two metaphase chromosomes showing spindle microtubule associated with the two kinetochores. Fewer gold particles are found at the kinetochore (arrowhead). In some cases, virtually no gold particles are associated with kinetochore (arrows). Examination of serial section revealed an essentially the same outcome. (c) Alterative view of two metaphase kinetochores connected with spindle microtubules. While no gold particles are associated with the kinetochore (arrows) majority of gold particle deposition is seen on spindle microtubules toward spindle poles (arrowheads). (d) Magnified view of spindle microtubules adjacent to centrioles. Abundance of gold particle labeling is readily apparent on spindle microtubules near the pole (arrowheads). Diminished deposition of gold particles at the kientochore of metaphase HeLa cells indicates that relocation of TTK from kinetochore aligned at the equator, a time marks spindle checkpoint silence. Bars: (a) $2 \mu \mathrm{m}$; (b-d) $100 \mathrm{~nm}$.

there were great amount of $10 \mathrm{~nm}$ gold particles adjacent to the spindle microtubules away from the kinetochore (Fig $3 \mathrm{c}$ and $3 \mathrm{~d}$, arrowheads). Serial sections revealed no TTK labeling on the sister kinetochores of fully congressed chromosomes. This is consistent with 


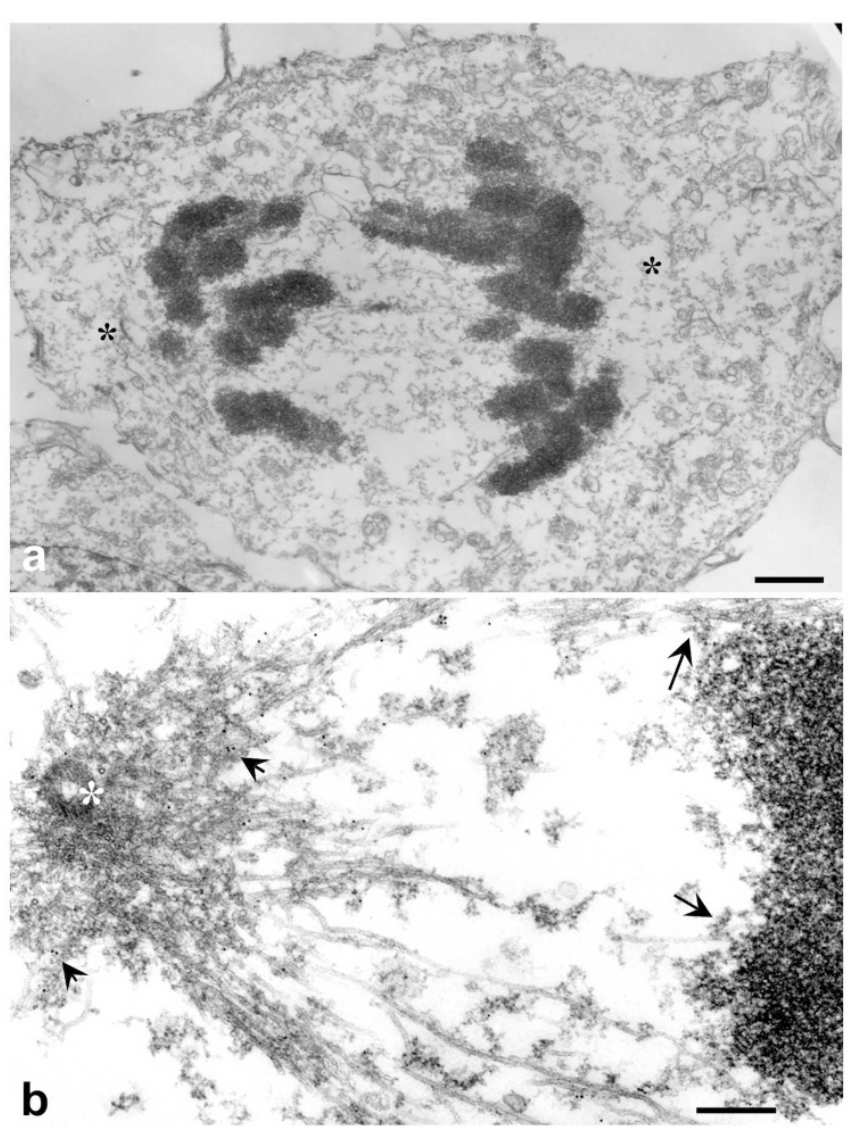

Fig 4. TTK dissociates from kinetochore after sister chromatids separation. HeLa cells were processed as described in Materials and Methods. (a) Low magnification view of an anaphase HeLa cell. The two spindle pole positions are marked with asterisks. (b) Magnified view of a kinetochore-microtubule interface shows that no gold particles are seen in the kinetochore (arrowheads). However, some gold particles are found around the centrosome (arrow). Examination of the number of particles revealed a reduction relative to metaphase. Bars: (a) $2 \mu \mathrm{m}$; (b) $300 \mathrm{~nm}$.

the previously reported position of other spindle checkpoint components such as Mad2[27]. Thus, TTK is located in the outer surface of the kinetochores of prometaphase chromosome and is dissociated upon the metaphase alignment.

\section{TTK is no longer associated with kinetochore after sister chromatids separate}

To verify if TTK is located in the kinetochore corona during anaphase chromosome movement toward the poles, TTK positioning was examined in an anaphase cell (Fig 4a-b). There is no gold particle deposition found when serial sections of the separated sister kinetochores were examined. Later in anaphase, when chromosomes have moved close to the poles, and pole elongation has been initiated (anaphase B); no TTK is found to be associated with the kinetochore (data not shown). Examination of 13 anaphase kinetochore sections (from both randomly picked and serial stacks) revealed no gold particles was associated with the kinetochores (Fig 4b, arrows). However, there were gold particles found around spindle poles (Fig 4b, arrowheads) with reduced abundance. Thus, we reasoned that TTK departed from the kinetochore prior to sister chromatid separation.

\section{DISCUSSION}

From these ultrastructural studies, we can develop a kinetic picture of TTK integration into kinetochore functional dynamics. The evidence presented here provides proof that TTK is re-distributed to develop kinetochores of condensing chromosomes from nuclear pore adjacent complex after initial nuclear envelope disassemble (Fig 2 ). In prometaphase, TTK binds along the surface of mono-oriented kinetochores that are attached to spindle microtubules in addition to spindle poles (Fig 2b, $\mathrm{d}$ and e). During congression, at metaphase, TTK is a component of kinetochore corona fibers, extending from the kinetochore outer plate (Fig 2c) and gradually dissociating from kinetochore upon the metaphase alignment. In addition, TTK is also located on spindle pole microtubules and poleward-translocating vesicles. The collective evidence strongly implicates $T T K$ is an essential spindle checkpoint gene that governs the integrity of mitotic spindle and kinetochore assembly dynamics.

\section{A model of TTK in spindle checkpoint signaling}

Our finding that TTK extends at least $50 \mathrm{~nm}$ from the kinetochore outer surface (modeled in Fig 5) reinforces several lines of evidence showing that altering TTK kinases participate in spindle checkpoint signaling: genetic disruption of TTK homologue MPS1 results in mitotic arrest[28, 29], antibodies to Xenopus MPS1 abrogates the spindle checkpoint in egg extracts and librates CENPE, Mad1 and Mad2 from kinetochore[24]. In addition, suppression of TTK expression in U2OS cells results in defects in spindle checkpoint[18]. Moreover, mass spectrometric identification of molecular composition of CENP-E complex revealed an association between TTKand CENP-E[17]. The initial transition of an apparent TTK complex toward the developing kinetochore at earliest prometaphase, is presumably mediated by 


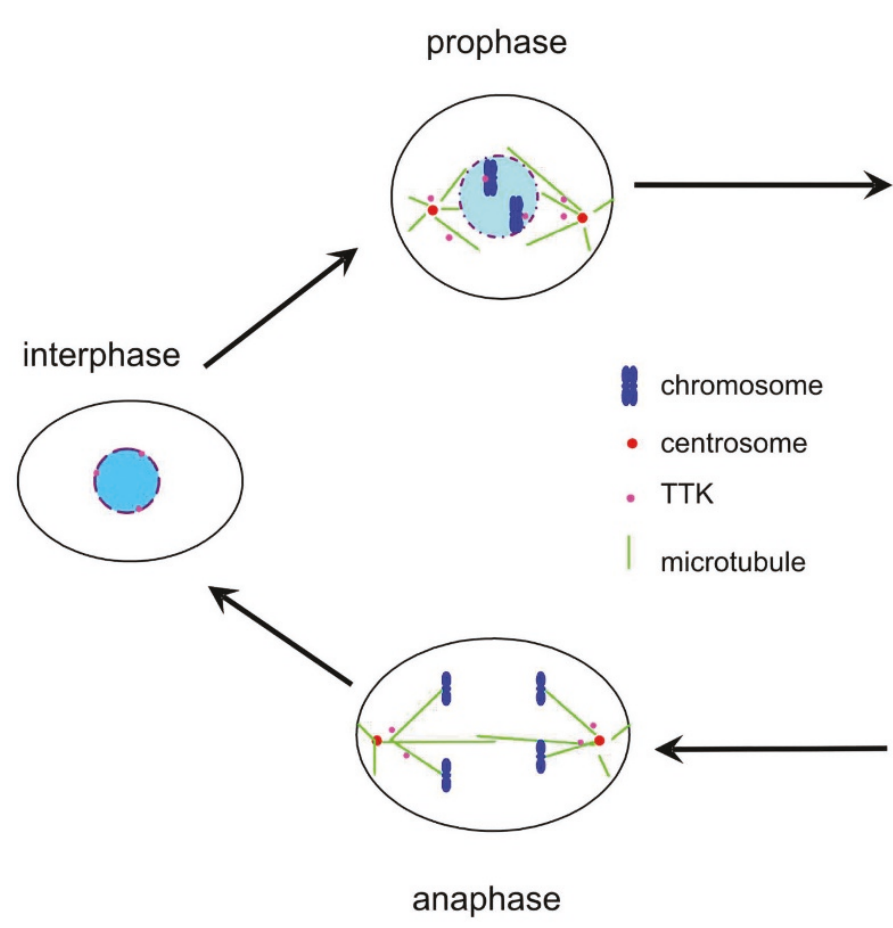

prometaphase
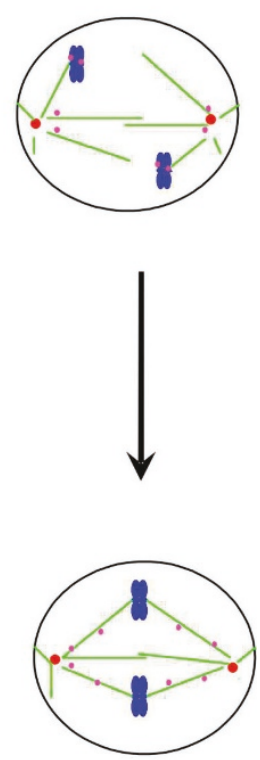

metaphase
Fig 5. Working model for TTK function in mitosis. TTK, denoted as purple dots, is located near the nuclear pore in the interphase cells and is recruited to the developing kinetochore and spindle poles (labeled in red) as the nuclear envelope disassembles. TTK situates on the outermost surface of mono-oriented kinetochore and bi-oriented kinetochore of prometaphase chromosomes (marked in blue). Upon chromosomal alignment, tension across the sister kinetochores diminishes and TTK dissociates from kinetochore for silencing spindle checkpoint. Sister chromatids separate after spindle checkpoint is satisfied. TTK then migrates toward the poles to exert its centrosome-based function.
CENP-E[17]. The initial transition of an apparent TTK complex toward the developing kinetochore at earliest prometaphase, is presumably mediated by CENP-E's plus end motor activity as TTK is co-purified with CENP-E form the prometaphase Hela lysates[17]. Interestingly, TTK departs from the kinetochore aligned at the equator (Fig 3). Given the fact that CENP-E has been shown to have the capacity to couple cargoes to microtubule depolymerization-driven, minus end-directed movement [30], it is plausible by analogy that poleward translocation of TTK may also tie to its association with CENPE. The centrosomal TTK may regulate spindle integrity and dynamics via phosphorylation of MAPs or other proteins involved in centrosomal duplication. During the preparation of this study, Liu et al (2003) have reported that human MPS1 is located at nuclear pore of interphase nucleus and to kinetochore and centrosome of mitotic cells[26]. Given the fact that the temporal order of dissociation of TTK from the kinetochore is correlated with the dephosphorylation profile of TTK reported by Liu et al[26], it is then possible that the phosphorylation of TTK may account for its function at the kinetochore. Further functional analysis will be required in order to distinguish between the functional roles of TTK in kinetochore and centrosome.

One important question addressed by our studies is the dynamic distribution of TTK during mitosis and its precise localization relative to the kinetochore. To these earlier research efforts, our finding that localization of TTK to the corona fibers of kinetochore concurrent with the release of other spindle checkpoint component such as $\operatorname{Mad} 2[27,31]$, together with its association with CENP-E implicates regulation of TTK-CENP-E association is an important tension sensor to the spindle microtubule-kinetochore associated mitotic checkpoint. In sum, our study strongly supports a model in which TTK functions in mitotic checkpoint signaling at both kinetochore and centrosome locations.

\section{ACKNOWLEDGEMENTS}

We thank members of our group for insightful discussion during the course of this study. This work was supported by grants from the Chinese Outstanding Young Scientist Award (39925018), the Chinese Academy of Science (KSCX2-2-01), the Chinese 973 project (2002CB713700), and the American Cancer Society (RPG59282) to XY.

\section{REFERENCES}

1 Cleveland DW, Mao Y, Sullivan KF. Centromeres and kinetochoresfrom epigenetics to mitotic checkpoint signaling. Cell 2003; 112:407-21.

2 Luykx P. The structure of the kinetochore in meiosis and mitosis 
Urechis eggs. Exp Cell Res 1965; 39:643-57.

3 Brinkley BR, Stubblefield E. The fine structure of the kinetochore of a mammalian cell in vitro. Chromosoma (Berl) 1966; 19:28-43

4 Ris H, Witt PL. Structure of the mammalian kinetochore. Chromosoma (Berl) 1981; 82:153-70.

5 Rattner JB. Organization within the mammalian kinetochore. Chromosoma (Berl.). 1986; 93:515-20.

6 Wordeman L, Mitchison TJ. Identification and partial characterization of mitotic centromere-associated kinesin, a kinesin-related protein that associates with centromeres during mitosis. J. Cell Biol 1995; 128:95-104.

7 Yao X, Anderson KL, Cleveland DW. The microtubule-depen dent motor centromere-associated protein E (CENP-E) is an integral component of kinetochore corona fibers that link centromeres to spindle microtubules. J Cell Biol 1997; 139: 43547.

8 Jokelainen PT. The ultrastructure and spatial organization of the metaphase kinetochore in mitotic rat cells. J Ultrastruct Res 1967; 19:19-44.

9 Comings DE, Okada TA. Holocentric chromosomes in oncopettus kinetochore plates are present in mitosis but absent in meiosis. Chromosoma (Berl.) 1973; 37:177-92.

10 Roos U-P. Light and electron microscopy of rat kangaroo cells in mitosis. I. Formation and breakdown of the mitotic apparatus. Chromosoma (Berl) 1973; 40:43-82.

11 Rieder CL. The formation, structure and composition of the mammalian kinetochore and kinetochore fiber. Int Rev Cytol 1982; 79:1-58.

12 McEwen BF, Arena JT, Frank J et al. Structure of the colcemidtreated PtK1 kinetochore outer plate as determined by high voltage electron microscopic tomography. J Cell Biol 1993;120:301-12.

13 Palmer DK, O'Day K, Trong HL et al. Purification of the centromere-specific protein CENP-A and demonstration that it is a distinctive histone. Proc Natl Acad Sci USA 1991; 88: 3734 8

14 Cooke CA, Bernat RL, Earnshaw WC. CENP-B: a major human centromere protein located beneath the kinetochore. J Cell Biol 1990; 110:1475-88.

15 Rattner JB, Rao A, Fritzler MJ et al. CENP-F is a .ca $400 \mathrm{kDa}$ kinetochore protein that exhibits a cell-cycle dependent localization. Cell Motil Cytoskeleton 1993; 26:214-26

16 Jablonski SA, Chan GK, Cooke CA et al. The hBUB1 and hBUBR1 kinases sequentially assemble onto kinetochores during prophase with hBUBR1 concentrating at the kinetochore plates in mitosis. Chromosoma 1998; 107:386-96.

17 Zhang J, Fu C, Miao Y et al. Protein kinase TTK interacts and co-localizes with CENP-E to the kinetochore of human cells. Sci Bull 2002; 27:213-9.

18 Stucke VM, Sillje HH, Arnaud L et al. Human Mps1 kinase is required for the spindle assembly checkpoint but not for centrosome duplication. EMBO J 2002; 21:1723-32.

19 Mills GB, Schmandt R, McGill M et al. Expression of TTK, a novel human protein kinase, is associated with cell proliferation. J Biol Chem 1992; 267:16000-6.

20 Hogg D, Guidos C, Bailey D et al. Cell cycle dependent regulation of the protein kinase TTK. Oncogene 1994; 9:89-96.

21 Fisk HA, Winey M. The mouse Mps1p-like kinase regulates centrosome duplication. Cell 2001; 106:95-104.

22 Lindberg RA, Fischer WH, Hunter T. Characterization of a human protein threonine kinase isolated by screening an expression library with antibodies to phosphotyrosine. Oncogene 1993; 8: 351-9.

23 Lauze E, Stoelcker B, Luca FC et al. Yeast spindle pole body duplication gene MPS1 encodes an essential dual specificity protein kinase. EMBO J 1995; 14:1655-63.

24 Abrieu A, Magnaghi-Jaulin L, Kahana JA et al. Mps1 is a kinetochore-associated kinase essential for the vertebrate mitotic checkpoint. Cell 2001; 106: 83-93.

25 Yao X, Cheng L, Forte JG. Biochemical characterization of ezrinactin interaction. J Biol Chem 1996; 271:7224-9.

26 Liu ST, Chan GK, Hittle JC et al, Human MPS1 kinase is required for mitotic arrest induced by loss of CENP-E from kinetochores. Mol Biol Cell 2003; 14:1638-51.

27 Li Y, Benezra R. Identification of a human mitotic checkpoint gene: hsMAD2. Science (Wash DC) 1996; 274:246-9.

28 Hardwick KG, Weiss E, Luca FC et al. Activation of the bud-ding yeast spindle assembly checkpoint without mitotic spindle disruption. Science (Wash DC) 1996; 273:953-6.

29 Weiss E, Winey M. The Saccharomyces cerevisiae spindle pole body duplication gene MPS1 is part of a mitotic check-point. J Cell Biol 1996; 132:111-23.

30 Lombillo VA, Nislow C, Yen TJ et al. Antibodies to the kine sin motor domain and CENP-E inhibit microtubule depolymerization-dependent motion of chromosomes in vitro. J Cell Biol 1995; 128:107-15.

31 Chen R-H, Waters JC, Salmon ED et al. Association of spindle assembly checkpoint component XMAD2 with unattached kinetochores. Science (Wash DC) 1996; 274:242-6. 